T. Caraballo, K. Liu and X. Mao

Nagoya Math. J.

Vol. 161 (2001), 155-170

\title{
ON STABILIZATION OF PARTIAL DIFFERENTIAL EQUATIONS BY NOISE
}

\author{
TOMÁS CARABALLO, KAI LIU AND XUERONG MAO
}

\begin{abstract}
Some results on stabilization of (deterministic and stochastic) partial differential equations are established. In particular, some stability criteria from Chow [4] and Haussmann [6] are improved and subsequently applied to certain situations, on which the original criteria commonly do not work, to ensure almost sure exponential stability. This paper also extends to infinite dimension some results due to Mao [9] on stabilization of differential equations in finite dimension.
\end{abstract}

\section{$\S 1$. Introduction}

In this work, we shall present an investigation to stabilization of stochastic partial differential equations by noise. In order to motivate our theory, let us firstly analyze the following example from Haussmann [6].

Consider a one-dimensional rod of length $\pi$ whose ends are maintained at $0^{\circ}$ and whose sides are insulated. Assume that there is an exothermic reaction taking place inside the rod with heat being produced proportionally to the temperature. The temperature in the rod may be modeled to satisfy

$$
\left\{\begin{array}{l}
\frac{\partial X}{\partial t}=\frac{\partial^{2} X}{\partial y^{2}}+r X, \quad t>0, \quad 0<y<\pi \\
X(t, 0)=X(t, \pi)=0 \\
X(0, y)=x_{0}(y)
\end{array}\right.
$$

where $r$ depends on the rate of reaction. If we assume $r=r_{0}$, a constant, then we can solve

$$
X(t, y)=\sum_{n=1}^{\infty} a_{n} e^{-\left(n^{2}-r_{0}\right) t} \sin n y
$$

Received March 9, 1999. 
where $x_{0}(y)=\sum_{n=1}^{\infty} a_{n} \sin n y$. Hence we obtain exponential stability if $n^{2}>r_{0}$ for all $n \in \mathbf{N}$, or equivalently, $r_{0}<1$. Observe that, in general, for $r_{0} \geq 1$ the trivial solution is not stable.

Suppose now that $r$ is random, and assume it is modeled as $r_{0}+r_{1} \dot{w}$, so that the equation becomes

$$
d X=\left(\frac{\partial^{2}}{\partial y^{2}}+r_{0}\right) X d t+r_{1} X d w
$$

where $w$ is a one-dimensional Wiener process. Haussmann proved in [6] that when $r_{0}<1$ (i.e. the unperturbed system is very stable), the perturbed system $(*)$ remains pathwise exponentially stable if $r_{1}^{2}<2\left(1-r_{0}\right)$, i.e., if the perturbation is sufficiently small so that this relation is satisfied. On the other hand, since a new noise term is involved in the perturbed system $(*)$, a natural problem now arises: as $r_{0}>1$ (a case where Haussmann's results fail to be applied), is it possible to deduce any exponential stability results for the perturbed system? As we are going to see, by virtue of some general stabilization results to be derived in Section 3 below, we can actually prove that for arbitrary $r_{0} \in \mathbf{R}^{1}$, if $r_{1}^{2}>2\left(r_{0}-1\right)$, the perturbed system becomes exponentially stable. In other words, the multiplicative noise now plays the role to stabilize unstable deterministic systems. It is also worth pointing out that if $r_{0}<1$, by employing our results we deduce meanwhile that for arbitrary $r_{1} \in \mathbf{R}^{1}$, the system $(*)$ is still almost sure exponentially stable. This result greatly improves the original one in Haussmann [6].

Another more important fact we want to mention is that noise can also be used to stabilize some stochastic partial differential equations. Indeed, in the previous situation, i.e., if $r_{0}>1$ and $r_{1} \in \mathbf{R}^{1}$ satisfies $r_{1}^{2} \leq 2\left(r_{0}-1\right)$, we do not know whether the trivial solution is stable or not. But, if the system is perturbed by another multiplicative noise of the same type, say $r_{2} X \dot{w}_{1}$, where $w_{1}$ is another one-dimensional Wiener process independent of $w$, as a consequence of our main result (Theorem 4.1), it can be deduced that the system

$$
(* *) \quad d X=\left(\frac{\partial^{2}}{\partial y^{2}}+r_{0}\right) X d t+r_{1} X d w+r_{2} X d w_{1},
$$

becomes once more pathwise exponentially stable if $r_{2}$ is chosen large enough.

There existed some work on stabilization of stochastic differential equations by noise in finite dimension. The first one is due to Has'minskii [5], 
who stabilized a system by using two white noise sources. Later, Arnold et al. [1] showed, in particular, that the system $\dot{x}(t)=A x(t)$ can be stabilized by zero mean stationary parameter noise if and only if trace $A<0$. On the other hand, in the nonlinear case, Scheutzow [11] provided some examples on stabilization and destabilization in the plane, and Mao [9] obtained some results on stabilization and destabilization by Brownian motion for certain class of nonlinear stochastic differential equations. However, as far as we know, there existed no papers devoting themselves to the investigation of the analogous problems in infinite dimension, that is, stabilization of (nonlinear) partial differential equations. In this paper, we shall fill this gap and meanwhile improve some stability criteria in Caraballo and Liu [3], Chow [4] and Haussmann [6].

The organization of this paper is as follows. In Section 2, we shall prove some results in order to stabilize systems modeled by deterministic partial differential equations. Section 3 is devoted to applying the results obtained in Section 2 to analyze, in particular, the exponential stability of a stochastic nonlinear monotone equation which improves a stability result from Caraballo and Liu [3] and Chow [4]. Finally, we shall establish a stabilization result for stochastic partial differential equations in Section 4 which permits us to stabilize the stochastic systems studied in Section 3.

\section{$\S 2$. Stabilization of deterministic partial differential equations}

First of all, we introduce the framework where our analysis is going to be carried out.

Let $H$ be a real, separable Hilbert space and $V$ a real, reflexive and separable Banach space such that

$$
V \hookrightarrow H \equiv H^{\prime} \hookrightarrow V^{\prime}
$$

where the injections are continuous and dense. In particular, we also assume both $V$ and $V^{\prime}$ are uniformly convex.

We denote by $\|\cdot\|,|\cdot|$ and $\|\cdot\|_{*}$ the norms in $V, H$ and $V^{\prime}$ respectively; by $\langle\cdot, \cdot\rangle$ the duality product between $V, V^{\prime}$, and by $(\cdot, \cdot)$ the scalar product in $H$. Let $\beta$ be the constant of the injection $V \hookrightarrow H$, i.e.

$$
|x| \leq \beta\|x\|, \quad \forall x \in V .
$$

Consider the following problem

$$
\left\{\begin{array}{l}
d x(t)=F(t, x(t)) d t \\
x(0)=x_{0} \in H
\end{array}\right.
$$


where $F(t, \cdot): V \rightarrow V^{\prime}, t \in \mathbf{R}_{+}$, is a family of (nonlinear) operators satisfying $F(t, 0)=0$ and the following hypothesis:

There exist a continuous function $\nu(\cdot)$ and a real number $\nu_{0} \in \mathbf{R}^{1}$ such that

$$
2\langle x, F(t, x)\rangle \leq \nu(t)|x|^{2}, \quad \forall x \in V
$$

where

$$
\limsup _{t \rightarrow \infty} \frac{1}{t} \int_{0}^{t} \nu(s) d s \leq \nu_{0} .
$$

Assume that for each $x_{0} \in H$ there exists a unique strong solution to problem $(1), x\left(t ; x_{0}\right) \in L^{2}(0, T ; V) \cap C(0, T ; H)$. Observe that, when $F(t, \cdot)$ satisfies a coercivity condition of the type

$$
2\langle x, F(t, x)\rangle \leq-\varepsilon\|x\|^{p}+\alpha|x|^{2}, \quad \varepsilon>0, \alpha \in \mathbf{R}^{1}, p>1,
$$

and a monotonicity hypothesis, there exists a unique strong solution to (1) in $L^{p}(0, T ; V) \cap C(0, T ; H)$ (see, for instance, Lions [7]).

Note that this coercivity assumption obviously implies (2).

Now we can propose the following question: If problem (1) is not stable, can it be stabilized by using a stochastic perturbation of the type $g(t, x(t)) d w(t)$ ? Here $w(t)$ is (for simplicity) a standard real Wiener process defined on a certain complete probability space $(\Omega, \mathcal{F}, P)$ with filtration $\left(\mathcal{F}_{t}\right)_{t \geq 0}$, and $g(t, \cdot): H \rightarrow H$ satisfies $g(t, 0)=0$ and the following condition

$$
|g(t, x)-g(t, y)|^{2} \leq \lambda(t)|x-y|^{2}, \quad t \in \mathbf{R}_{+}, x, y \in H
$$

where $\lambda(\cdot)$ is a nonnegative continuous function such that

$$
\limsup _{t \rightarrow \infty} \frac{1}{t} \int_{0}^{t} \lambda(s) d s \leq \lambda_{0} \in \mathbf{R}_{+} .
$$

The answer to this question is affirmative for a suitably chosen $g$. Indeed, consider the following perturbed problem

$$
\left\{\begin{array}{l}
d u(t)=F(t, u(t)) d t+g(t, u(t)) d w(t), \quad t>0 \\
u(0)=u_{0} \in H .
\end{array}\right.
$$

As we are mainly interested in stability properties of the trivial solution to problem (7), we suppose that for each $u_{0} \in H$ there exists a unique strong solution to $(7)$ in $I^{p}(0, T ; V) \cap L^{2}(\Omega ; C(0, T ; H))$ for all $T>0$, and certain $p>1$, where $I^{p}(0, T ; V)$ denotes the space of all $V$-valued processes 
$u(t)$, measurable and satisfying $E \int_{0}^{T}\|u(t)\|^{p} d t<\infty$. (See, for instance, Pardoux [10] or Caraballo [2] for conditions under which there exists such a unique solution for initial values $u_{0} \in L^{2}\left(\Omega, \mathcal{F}_{0}, P ; H\right)$ )

Assume $V(t, x): \mathbf{R}_{+} \times H \rightarrow \mathbf{R}_{+}$is a $C^{1,2}$-positive functional such that for any $x \in V, t \in \mathbf{R}_{+}, V_{x}^{\prime}(t, x) \in V$. We define operators $L$ and $Q$ as follows: for $x \in V, t \in \mathbf{R}_{+}$

$$
L V(t, x)=V_{t}^{\prime}(t, x)+<V_{x}^{\prime}(t, x), F(t, x)>+\frac{1}{2}\left(V_{x x}^{\prime \prime}(t, x) g(t, x), g(t, x)\right)
$$

and

$$
Q V(t, x)=\left(V_{x}^{\prime}(t, x), g(t, x)\right)^{2}
$$

THEOREM 2.1. Assume the solution of (7) satisfies that $|u(t)| \neq 0$ for all $t \geq 0$ a.s. provided $\left|u_{0}\right| \neq 0$ a.s. Let $V(x) \in C^{2}\left(H ; \mathbf{R}_{+}\right)$and $\psi_{1}(t) \in \mathbf{R}^{1}$, $\psi_{2}(t) \geq 0$ be two continuous functions. Assume that there exist constants $p>0, \gamma \geq 0$ and $\theta \in \mathbf{R}^{1}$ such that

(a) $|x|^{p} \leq V(x), \quad \forall x \in V$;

(b) $\quad L V(t, x) \leq \psi_{1}(t) V(x), \quad \forall x \in V, \quad \forall t \in \mathbf{R}_{+}$;

(c) $\quad Q V(t, x) \geq \psi_{2}(t) V^{2}(x), \quad \forall x \in V, \quad \forall t \in \mathbf{R}_{+}$;

(d) $\quad \limsup _{t \rightarrow \infty} \frac{\int_{0}^{t} \psi_{1}(s) d s}{t} \leq \theta, \quad \liminf _{t \rightarrow \infty} \frac{\int_{0}^{t} \psi_{2}(s) d s}{t} \geq 2 \gamma$.

Then the strong solution of equation (7) satisfies

$$
\limsup _{t \rightarrow \infty} \frac{\log \left|u\left(t, u_{0}\right)\right|}{t} \leq-\frac{\gamma-\theta}{p} \text { a.s. }
$$

where $u_{0} \in H$ is an $\mathcal{F}_{0}$-measurable random vector such that $\left|u_{0}\right| \neq 0$ a.s. In particular, if $\gamma>\theta$, the solution is almost sure exponentially stable.

Proof. Fix $u_{0} \in H$ such that $\left|u_{0}\right| \neq 0$ a.s. It is easy to deduce by Itô's formula that

$$
\begin{aligned}
\log V\left(u\left(t, u_{0}\right)\right) \leq & \log V(u(0))+M(t) \\
& +\int_{0}^{t}\left(\frac{L V(s, u(s))}{V(u(s))}-\frac{1}{2} \frac{Q V(s, u(s))}{V^{2}(u(s))}\right) d s
\end{aligned}
$$

where $M(t)=\int_{0}^{t} \frac{1}{V(u(s))}\left(V_{x}^{\prime}(u(s)), g(s, u(s))\right) d w(s)$. 
By applying the exponential martingale inequality (e.g., see Lemma 1.1 in [8]), we can deduce

$$
P\left\{\omega: \sup _{0 \leq t \leq w}\left[M(t)-\int_{0}^{t} \frac{u}{2} \frac{1}{V^{2}(u(s))} Q V(s, u(s)) d s\right]>v\right\} \leq e^{-u v}
$$

for any positive constants $u, v$ and $w$. Assigning $\epsilon>0$ arbitrarily and taking

$$
u=\alpha, \quad v=2 \alpha^{-1} \log k, \quad w=k \epsilon, \quad k=1,2,3, \ldots,
$$

where $0<\alpha<1$, we then apply the well-known Borel-Cantelli lemma to obtain that there exists an integer $k_{0}(\epsilon, \omega)>0$ for almost all $\omega \in \Omega$ such that

$$
M(t) \leq 2 \alpha^{-1} \log k+\frac{\alpha}{2} \int_{0}^{t} \frac{Q V(s, u(s))}{V^{2}(u(s))} d s
$$

for all $0 \leq t \leq k \epsilon, k \geq k_{0}(\epsilon, \omega)$. Substituting this into (9) and using conditions $(\mathrm{b})(\mathrm{c})$, we see that there exists a positive random integer $k_{1}(\epsilon)$ such that almost surely

$\log V(u(t)) \leq \log V(u(0))+2 \alpha^{-1} \log k+\int_{0}^{t} \psi_{1}(s) d s-\frac{1}{2}(1-\alpha) \int_{0}^{t} \psi_{2}(s) d s$

for all $(k-1) \epsilon \leq t \leq k \epsilon, k \geq k_{0}(\epsilon, \omega) \vee k_{1}(\epsilon)$ which for the preceding $\epsilon>0$, together with $(\mathrm{d})$, immediately implies that

$$
\begin{aligned}
\frac{\log |u(t)|}{t} & \leq \frac{\log V(u(t))}{p t} \\
& \leq \frac{1}{p t}\left(\log V(u(0))+2 \alpha^{-1} \log k+(\theta+\epsilon) t-\frac{1}{2}(1-\alpha)(2 \gamma+\epsilon) t\right) .
\end{aligned}
$$

Therefore,

$$
\limsup _{t \rightarrow \infty} \frac{\log |u(t)|}{t} \leq \frac{1}{p}\left[(\theta+\epsilon)-(1-\alpha)\left(\gamma+\frac{\epsilon}{2}\right)\right] \quad \text { a.s. }
$$

Letting $\alpha \rightarrow 0, \epsilon \rightarrow 0$, we can immediately obtain

$$
\limsup _{t \rightarrow \infty} \frac{\log \left|u\left(t, u_{0}\right)\right|}{t} \leq-\frac{\gamma-\theta}{p} \quad \text { a.s. }
$$

As a direct consequence, we immediately obtain the following: 
Theorem 2.2. Assume the solution of (7) satisfies that $\left|u\left(t, u_{0}\right)\right| \neq 0$ for all $t \geq 0$ almost surely provided $\left|u_{0}\right| \neq 0$ almost surely. In addition to hypotheses (2), (3), (5) and (6), assume the following one holds:

$$
(g(t, x), x)^{2} \geq \rho(t)|x|^{4}, \quad \forall x \in H,
$$

where $\rho(\cdot)$ is a nonnegative continuous function such that

$$
\liminf _{t \rightarrow \infty} \frac{1}{t} \int_{0}^{t} \rho(s) d s \geq \rho_{0}, \quad \rho_{0} \in \mathbf{R}_{+} .
$$

Then, if $u\left(t, u_{0}\right)$ denotes the solution to (7), it follows

$$
\limsup _{t \rightarrow \infty} \frac{1}{t} \log \left|u\left(t, u_{0}\right)\right|^{2} \leq-\left(2 \rho_{0}-\nu_{0}-\lambda_{0}\right), \quad P-a . s .
$$

for any $u_{0} \in H$. In particular, if $2 \rho_{0}>\nu_{0}+\lambda_{0}$ the equation (7) is almost surely exponentially stable.

Proof. The proof follows immediately from Theorem 2.1 by setting $V(t, x)=|x|^{2}, x \in H$.

Remarks. 1. Observe that this result can be interpreted as follows. Given the deterministic system (1) satisfying (2)-(3), if a stochastic multiplicative noise, say $g(t, u) \dot{w}(t)$, appears in the system, the perturbed one (7) becomes exponentially stable with probability one (w.p.1 in the sequel) for suitable $g$. In particular, one can always stabilize the system (1) by considering linear perturbations of the type $g(t, u)=\rho_{0} u, \rho_{0} \in \mathbf{R}^{1}$.

2. An analogous result holds when $g(t, \cdot): V \rightarrow H$, assuming the following condition instead of (2)-(6):

$$
2\langle x, F(t, x)\rangle+|g(t, x)|^{2} \leq \delta(t)|x|^{2}, \quad t \in \mathbf{R}_{+}, x \in V,
$$

where $\delta(\cdot)$ is a nonnegative continuous function such that

$$
\limsup _{t \rightarrow \infty} \frac{1}{t} \int_{0}^{t} \delta(s) d s \leq \delta_{0} \in \mathbf{R} .
$$

In this case, (11) is replaced by

$$
\limsup _{t \rightarrow \infty} \frac{1}{t} \log \left|u\left(t, u_{0}\right)\right|^{2} \leq-\left(2 \rho_{0}-\delta_{0}\right), \quad P-a . s .
$$




\section{$\S 3$. Applications}

\subsection{A linear example}

First of all, let us consider the simple linear case described in the beginning of Section 1. We put this problem into our formulation by setting $V=H_{0}^{1}([0, \pi]), H=L^{2}([0, \pi]), F(t, u)=\frac{\partial^{2} u}{\partial y^{2}}+r_{0} u, u \in V, g(t, u)=$ $r_{1} u, u \in H$.

Now, it is easy to check that Theorem 2.2 can be applied to this problem with $\rho_{0}=\lambda_{0}=r_{1}^{2}$ and $\nu_{0}=2\left(r_{0}-1\right)$. Thus, one can obtain for the solutions to $(*)$

$$
\limsup _{t \rightarrow+\infty} \frac{1}{t} \log |X(t)| \leq-\left(r_{1}^{2}-2\left(r_{0}-1\right)\right)
$$

with probability 1 . In other words, we get pathwise exponential stability if $r_{1}^{2}>2\left(r_{0}-1\right)$.

Observe that, in particular, when $r_{0}<1$, the trivial solution to Eq. $(*)$ is $P$-almost surely exponentially stable for all $r_{1} \in \mathbf{R}$, which greatly improves the results obtained in Haussmann [6] since the restriction imposed on $r_{1}$ is not necessary here.

\subsection{A nonlinear example}

Now we are going to apply Theorem 2.2 to analyze the pathwise stability of a nonlinear stochastic partial differential equation (which contains the first example as a special case). Consider the following problem previously studied, among others, by Pardoux [10], Caraballo and Liu [3] (and also by Chow [4] in the autonomous case):

$$
\left\{\begin{array}{l}
d u(t)=A(t, u(t)) d t+B(t, u(t)) d w(t) \\
u(0)=u_{0} \in H
\end{array}\right.
$$

where $A(t, \cdot): V \rightarrow V^{\prime}$ is a family of nonlinear operators defined a.e.t. satisfying $A(t, 0)=0$ for all $t \in \mathbf{R}_{+} ; B(t, \cdot): V \rightarrow H$, satisfies

(b.1) $B(t, 0)=0$;

(b.2) There exists $k>0$ such that

$$
|B(t, y)-B(t, x)| \leq k\|y-x\|, \quad \forall x, y \in V \text {, a.e.t }
$$

In $[3]$ it is proved the following result:

THEOREM 3.1. In addition to (b.1)-(b.2), assume the following coercivity condition: 
There exist $\alpha>0, p>1$ and $\lambda \in \mathbf{R}^{1}$ such that for almost all $t \in \mathbf{R}_{+}$ and all $x \in V$,

$$
2\langle x, A(t, x)\rangle+|B(t, x)|^{2} \leq-\alpha\|x\|^{p}+\lambda|x|^{2} .
$$

Then, there exists $r>0$ such that

$$
E\left|u\left(t ; u_{0}\right)\right|^{2} \leq E\left|u_{0}\right|^{2} e^{-r t}, \quad \forall t \geq 0,
$$

if either one of the following hypotheses holds:

(i) $\lambda<0(\forall p>1)$;

(ii) $\lambda \beta^{2}-\alpha<0(p=2)$.

Furthermore, under the same assumptions the solution is almost surely exponentially stable. That is, there exist positive constants $\xi, \eta$ and a subset $\Omega_{0} \subset \Omega$ with $P\left(\Omega_{0}\right)=0$ such that, for each $\omega \notin \Omega_{0}$, there exists a positive random number $T(\omega)$ such that

$$
\left|u\left(t, \omega ; u_{0}\right)\right|^{2} \leq \eta\left|u_{0}\right|^{2} e^{-\xi t}, \quad \forall t \geq T(\omega) .
$$

Observe that, in the practical applications, conditions (i) and (ii) mean that the term containing $B$ must be small enough with respect to $A$. For example, let $\mathcal{O}$ be an open, bounded subset in $\mathbf{R}^{N}$ with regular boundary and let $2 \leq p<+\infty$. Consider the Sobolev spaces $V=W_{0}^{1, p}(\mathcal{O}), H=$ $L^{2}(\mathcal{O})$ with their usual inner products, and the monotone operator $A: V \rightarrow$ $V^{\prime}$ defined as

$$
\begin{aligned}
&\langle v, A u\rangle=-\sum_{i=1}^{N} \int_{\mathcal{O}}\left|\frac{\partial u(x)}{\partial x_{i}}\right|^{p-2} \frac{\partial u(x)}{\partial x_{i}} \frac{\partial v(x)}{\partial x_{i}} d x \\
&+\int_{\mathcal{O}} a u(x) v(x) d x \quad \forall u, v \in V
\end{aligned}
$$

where $a \in \mathbf{R}^{1}$. We also consider $B(t, u)=b u, u \in H$, where $b \in \mathbf{R}^{1}$. Finally, let $w(t)$ be a standard real Wiener process.

Then,

$$
2\langle x, A(t, x)\rangle+|B(t, x)|^{2}=-2\|x\|^{p}+2 a|x|^{2}+b^{2}|x|^{2}, \quad x \in V,
$$

so (16) holds with equality for $\alpha=2$ and $\lambda=2 a+b^{2}$. Now, condition (i) requires $2 a+b^{2}<0$, so $a<0$ and $b^{2}<-2 a$. On the other hand, (ii) will hold 
whenever $\left(2 a+b^{2}\right) \beta^{2}-2<0$, that is, $b^{2}<2 \beta^{-2}-2 a$. Therefore, Theorem 2.2 guarantees exponential stability of paths w.p.1 only for these values of $a$ and $b$ which means (as one can easily check) that the deterministic system $d u(t)=A(t, u(t)) d t$ is exponentially stable and the random perturbation is small enough. However, Theorem 2.2 ensures exponential stability for sufficiently large perturbations although the deterministic system is unstable. Note that, in this case, it is not difficult to see that

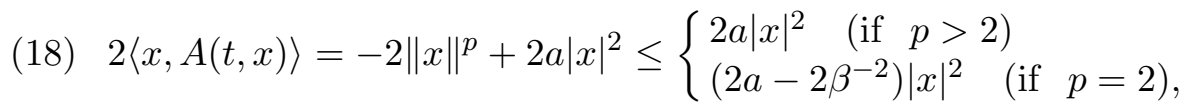
so, $\nu(t)=\nu_{0}=\left\{\begin{array}{l}2 a \quad(\text { if } p>2) \\ \left(2 a-2 \beta^{-2}\right) \quad(\text { if } p=2)\end{array}, \lambda_{0}=\rho_{0}=b^{2}\right.$, and then Theorem 2.2 yields

$$
\limsup _{t \rightarrow \infty} \frac{1}{t} \log \left|u\left(t ; u_{0}\right)\right|^{2} \leq\left\{\begin{array}{l}
-\left(b^{2}-2 a\right) \quad(\text { if } p>2) \\
-\left(b^{2}-2 a+2 \beta^{-2}\right) \quad(\text { if } p=2)
\end{array}\right.
$$

and, consequently, we get pathwise exponential stability w.p.1 if

$$
b^{2}>\left\{\begin{array}{l}
2 a \quad(\text { if } p>2) \\
2 a-2 \beta^{-2} \quad(\text { if } p=2)
\end{array}\right.
$$

In general, we can prove the following.

Theorem 3.2. Assume (b.1),(b.2),(16) and that there exists a nonnegative continuous function $b(\cdot)$ such that

$$
(B(t, x), x)^{2} \geq b(t)|x|^{4} \quad \forall x \in V
$$

with

$$
\liminf _{t \rightarrow+\infty} \frac{1}{t} \int_{0}^{t} b(s) d s \geq b_{0} \in \mathbf{R}_{+} .
$$

Then, P-a.s. it follows

$$
\limsup _{t \rightarrow+\infty} \frac{1}{t} \log \left|u\left(t ; u_{0}\right)\right|^{2} \leq\left\{\begin{array}{l}
-\left(2 b_{0}-\lambda\right) \quad(\text { if } p>1) \\
-\left(2 b_{0}-\lambda+\alpha \beta^{-2}\right) \quad(\text { if } p=2) .
\end{array}\right.
$$

for any $u_{0} \in L^{2}\left(\Omega, \mathcal{F}_{0}, P ; H\right)$ such that $\left|u_{0}\right| \neq 0, P$-a.s.

Proof. The proof easily follows from Theorem 2.2 and Remark 2 in Section 2. 
Remark. Observe that if $\lambda<0$, then (15) is pathwise exponentially stable w.p.1 for all $p>1$ and all $b_{0} \in \mathbf{R}_{+}$; while when $\lambda>0,(15)$ is stable if $2 b_{0}>\lambda$ (for $p \neq 2$ ) or $2 b_{0}>\lambda-\alpha \beta^{-2}$ (for $p=2$ ). Now, taking into account Theorem 2.2 and Theorem 3.1, let us summarize the analysis for the preceding particular example:

- Case 1: The nonlinear problem, i.e., $p>2$. Observe that in this case, the problem is exponentially stable for all $b \in \mathbf{R}^{1}$ when $a \leq 0$. However, if $a>0$ Theorem 2.2 gives stability provided $b^{2}>2 a$. Note that, we do not know what happens if $a>0$ and $b^{2} \leq 2 a$.

- Case 2: The linear problem, i.e., $p=2$. As in the preceding case, when $a \leq 0$ the system is almost surely exponentially stable for all $b \in \mathbf{R}^{1}$. But if $a>0$, we need check (ii) which requires $b^{2}<2 \beta^{-2}-2 a$, or it should hold $b^{2}>2 a-2 \beta^{-2}$. So, if $a \leq \beta^{-2}$ exponential stability w.p. 1 follows for all $b \in \mathbf{R}^{1}$. But, when $a>\beta^{-2}$, we only can ensure stability for $b^{2}>2 a-2 \beta^{-2}$ and we do not know what happens for $b^{2} \leq 2 a-2 \beta^{-2}$.

In conclusion, our analysis permits us, in particular, to guarantee exponential stability for a wide range of values of $a$ and $b$ where Theorem 3.1 can not be applied. Of course, this also means that, given the deterministic system $d x(t)=A(t, x(t)) d t$, if a stochastic perturbation of the type $b x(t) d w(t)$ appears, the perturbed systems become exponentially stable when the parameter of the noise satisfies the conditions above. But when this does not happen, that is, when we do not know whether the system is stable or not, what can we say? The answer motivating the following section is that the perturbed stochastic system once again can be stabilized by introducing another noise of the same type.

\section{$\S 4$. Stabilization of stochastic PDEs}

In the previous sections, we have already shown that stochastic perturbation can stabilize a deterministic partial differential equation. Now we shall prove that noise can also be used to stabilize stochastic partial differential equations.

Consider the system

$$
\left\{\begin{array}{l}
d x(t)=F(t, x(t)) d t+g(t, x(t)) d w_{1}(t)+h(t, x(t)) d w_{2}(t), t>0 \\
x(0)=x_{0} \in L^{2}\left(\Omega, \mathcal{F}_{0}, P ; H\right)
\end{array}\right.
$$


where $w_{1}$ and $w_{2}$ are two independent standard Wiener processes on the same probability basis, $F(t, \cdot): V \rightarrow V^{\prime}$ is a family of (nonlinear) operators with $F(t, 0)=0, g(t, \cdot), h(t, \cdot): H \rightarrow H$ are Lipschitz continuous, satisfying $g(t, 0)=h(t, 0)=0$ and the following assumptions:

There exists a continuous function $\tilde{\nu}(\cdot), t \geq 0$, and $\tilde{\nu}_{0} \in \mathbf{R}^{1}$ such that

$$
\limsup _{t \rightarrow \infty} \frac{1}{t} \int_{0}^{t} \tilde{\nu}(s) d s \leq \tilde{\nu}_{0}
$$

and

$$
2\langle x, F(t, x)\rangle+|g(t, x)|^{2} \leq \tilde{\nu}(t)|x|^{2}, \quad t \geq 0, \quad x \in V
$$

There exist nonnegative functions $\tilde{\lambda}(\cdot) \tilde{\rho}(\cdot), t \geq 0$, and $\tilde{\lambda}_{0}, \tilde{\rho}_{0} \in \mathbf{R}_{+}$such that

$$
\begin{gathered}
|h(t, x)|^{2} \leq \tilde{\lambda}(t)|x|^{2}, \quad t \geq 0, x \in H, \\
(x, h(t, x))^{2} \geq \tilde{\rho}(t)|x|^{4}, \quad t \geq 0, x \in H,
\end{gathered}
$$

where

$$
\limsup _{t \rightarrow \infty} \frac{1}{t} \int_{0}^{t} \tilde{\lambda}(s) d s \leq \tilde{\lambda}_{0}, \quad \liminf _{t \rightarrow \infty} \frac{1}{t} \int_{0}^{t} \tilde{\rho}(s) d s \geq \tilde{\rho}_{0}
$$

As in Section 2, we suppose that for each $u_{0} \in L^{2}\left(\Omega, \mathcal{F}_{0}, P ; H\right)$, there exists a unique strong solution to $(22)$ in $I^{p}(0, T ; V) \cap L^{2}(\Omega ; C(0, T ; H))$ for all $T>0$, and certain $p>1$. Observe that equation (22) can be regarded as the stochastically perturbed system of the following one

$$
\left\{\begin{array}{l}
d u(t)=F(t, u(t)) d t+g(t, u(t)) d w_{1}(t), t>0 \\
u(0)=x_{0} \in L^{2}\left(\Omega, \mathcal{F}_{0}, P ; H\right)
\end{array}\right.
$$

Now we can prove the following generalization of Theorem 2.2.

TheOrem 4.1. Assume hypotheses (9), (10), (23)-(27) hold. Then the strong solution of the equation (22), denoted by $x\left(t, x_{0}\right)$, satisfies

(29) $\quad \limsup _{t \rightarrow \infty} \frac{1}{t} \log \left|x\left(t, x_{0}\right)\right|^{2} \leq-\left(2\left(\rho_{0}+\tilde{\rho}_{0}\right)-\tilde{\nu}_{0}-\tilde{\lambda}_{0}\right), \quad P-$ a.s.

for any $x_{0} \in L^{2}\left(\Omega, \mathcal{F}_{0}, P ; H\right)$ such that $\left|x_{0}\right| \neq 0, \quad P$-a.s. In particular, if $2 \tilde{\rho}_{0}>\tilde{\nu}_{0}+\tilde{\lambda}_{0}-2 \rho_{0}$, the equation (22) is almost surely exponentially stable. 
Proof. Fix any $x_{0} \in L^{2}\left(\Omega, \mathcal{F}_{0}, P ; H\right)$ such that $\left|x_{0}\right| \neq 0, \quad P$-a.s. Then, in view of uniqueness and continuity of the solution, $|x(t)|:=\left|x\left(t, x_{0}\right)\right| \neq 0$ for all $t \geq 0 P$-a.s. On the other hand, by Itô's formula we have

$$
\begin{aligned}
\log |x(t)|^{2}= & \log \left|x_{0}\right|^{2}+\int_{0}^{t} \frac{2}{|x(s)|^{2}}\langle x(s), F(s, x(s))\rangle d s \\
& +\frac{1}{2} \int_{0}^{t}\left[\frac{2|g(s, x(s))|^{2}}{|x(s)|^{2}}-\frac{4(x(s), g(s, x(s)))^{2}}{|x(s)|^{4}}\right] d s \\
& +\frac{1}{2} \int_{0}^{t}\left[\frac{2|h(s, x(s))|^{2}}{|x(s)|^{2}}-\frac{4(x(s), h(s, x(s)))^{2}}{|x(s)|^{4}}\right] d s \\
& +\int_{0}^{t} \frac{2}{|x(s)|^{2}}(x(s), g(s, x(s))) d w_{1}(s) \\
& +\int_{0}^{t} \frac{2}{|x(s)|^{2}}(x(s), h(s, x(s))) d w_{2}(s)
\end{aligned}
$$

Since the last two terms in (31) are continuous martingales vanishing at $t=0$, it follows from the law of the iterated logarithm that

$$
\begin{aligned}
& \lim _{t \rightarrow+\infty} \frac{1}{t} \int_{0}^{t} \frac{2}{|x(s)|^{2}}(x(s), g(s, x(s))) d w_{1}(s)=0, \quad P-a . s . \\
& \lim _{t \rightarrow+\infty} \frac{1}{t} \int_{0}^{t} \frac{2}{|x(s)|^{2}}(x(s), h(s, x(s))) d w_{2}(s)=0, \quad P-a . s .
\end{aligned}
$$

Therefore, taking into account the hypotheses in the theorem we can get

$$
\limsup _{t \rightarrow+\infty} \frac{1}{t} \log |x(t)|^{2} \leq-\left[2\left(\rho_{0}+\tilde{\rho}_{0}\right)-\tilde{\nu}_{0}-\tilde{\lambda}_{0}\right], \quad P-a . s .
$$

Applications. As applications of this result we can conclude that for those examples in Section 3, whose stability properties are commonly not true, we can however stabilize those by introducing another stochastic perturbation.

More precisely, for the example modeled by Eq. $(* *)$, one can easily check that $\tilde{\nu}_{0}=2\left(r_{0}-1\right)+r_{1}^{2}, \tilde{\lambda}_{0}=\tilde{\rho}_{0}=r_{2}^{2}$ and $\rho_{0}=r_{1}^{2}$. So, (29) becomes

$$
\limsup _{t \rightarrow \infty} \frac{1}{t} \log \left|x\left(t, x_{0}\right)\right|^{2} \leq-\left(r_{1}^{2}+r_{2}^{2}-2\left(r_{0}-1\right)\right), \quad P-a . s .
$$


and, consequently, almost sure exponential stability follows if $r_{2}^{2}>2\left(r_{0}-\right.$ 1) $-r_{1}^{2}$.

On the other hand, consider the problem (15) and assume (b.1), (b.2), (16), (19), (20) hold. Suppose that another stochastic perturbation appears, i.e., consider

$$
\left\{\begin{array}{l}
d x(t)=A(t, x(t)) d t+B(t, x(t)) d w_{1}(t)+C(t, x(t)) d w_{2}(t) \\
x(0)=x_{0} \in L^{2}\left(\Omega, \mathcal{F}_{0}, P ; H\right)
\end{array}\right.
$$

where $C(t, \cdot): V \rightarrow H$ satisfies

(c.1) $C(t, 0)=0$;

(c.2) There exists $k>0$ such that

$$
|C(t, y)-C(t, x)| \leq k_{1}\|y-x\|, \quad \forall x, y \in V, \text { a.e.t }
$$

and there exists a nonnegative continuous function $c(\cdot)$ such that

$$
(C(t, x), x)^{2} \geq c(t)|x|^{4}, \quad \forall x \in V
$$

with

$$
\liminf _{t \rightarrow+\infty} \frac{1}{t} \int_{0}^{t} c(s) d s \geq c_{0} \in \mathbf{R}_{+} .
$$

Then,

(34) $\limsup _{t \rightarrow+\infty} \frac{1}{t} \log \left|x\left(t ; x_{0}\right)\right|^{2} \leq\left\{\begin{array}{l}-\left[2\left(b_{0}+c_{0}\right)-\lambda\right] \quad(\text { if } p>1) \\ \left.-\left[2\left(b_{0}+c_{0}\right)-\lambda+\alpha \beta^{-2}\right] \quad \text { (if } p=2\right) .\end{array}\right.$

So, for the situations where $2 b_{0}-\lambda<0$ (if $p \neq 2$ ) or $2 b_{0}-\lambda+\alpha \beta^{-2}<0$ (if $p=2$ ), we can always choose a linear perturbation $C(t, x)=c_{0}^{1 / 2} x$ with $c_{0}$ sufficiently large such that $2\left(b_{0}+c_{0}\right)-\lambda>0$ or $2\left(b_{0}+c_{0}\right)-\lambda+\alpha \beta^{-2}>0$ which imply that the perturbed system is almost surely exponentially stable.

Acknowledgements. The research of T. Caraballo has been partially supported by D.G.I.C.Y.T (Spain) Proyecto No. PB95-1242. K. Liu would also like to acknowledge the support of EPSRC Grant GR/K 70397 and X.R. Mao acknowledges the support of BBSRC grant 78/MM109712. 


\section{REFERENCES}

[1] L. Arnold, H. Crauel and V. Wihstutz, Stabilization of linear systems by noise, SIAM J. Control Optim., 21 (1983), 451-461.

[2] T. Caraballo, Existence and uniqueness of solutions for non-linear stochastic partial differential equations, Collect. Math., 42 (1) (1991), 51-74.

[3] T. Caraballo and K. Liu, On exponential stability criteria of stochastic partial differential equations, Stoch. Proc. Appl., 83 (1999), 289-301.

[4] P. L. Chow, Stability of nonlinear stochastic evolution equation, J. Math. Anal. Appl., 89 (2) (1982), 400-419.

[5] R.Z. Has'minskii, Stochastic Stability of Differential Equations, Sijthoff and Noordhoff, Alphen aan den Rijn, The Netherlands, 1980.

[6] U. G. Haussmann, Asymptotic Stability of the Linear Ito Equation in Infinite Dimensions, J. Math. Anal. Appl. (1978), 219-235.

[7] J.L. Lions, Quelque méthodes de résolution des problemes aux limites non lineaires, Dunod Gauthier-Villars, Paris, 1969.

[8] K. Liu, On stability for a class of semilinear stochastic evolution equations, Stoch. Proc. Appl., 70 (1997), 219-241.

[9] X. R. Mao, Stochastic stabilization and destabilization, Systems and Control Letts., 23 (1) (1994), 279-290.

[10] E. Pardoux, Equations aux Dérivées Partielles Stochastiques Nonlinéaires Monotones, Thesis, Université Paris Sud, 1975, pp. 279-290.

[11] M. Scheutzow, Stabilization and destabilization by noise in the plane, Stoch. Anal. Appl., 11 (1) (1993), 97-113.

Tomás Caraballo

Dpto. Ecuaciones Diferenciales y Análisis Numérico,

Universidad de Sevilla

Apartado de Correos 1160, 41080-Sevilla

Spain

caraball@cica.es

Kai Liu

Department of Mathematics, University of Wales Swansea,

Singleton Park, Swansea SA2 8PP

United Kingdom

k.liu@swansea.ac.uk

Current Address

Department of Probability and Statistics,

University of Sheffield

The Hicks Building, Sheffield S3 7RH

United Kingdom

K.Liu@sheffield.ac.uk 
Xuerong Mao

Department of Statistics and Modelling Science, University of Strathclyde, Glasglow G1 1XH, Scotland, United Kingdom

xuerong@stams.strath.ac.uk 